Die Wissenschaft von der Politik Band 4 
Die Wissenschaft von der Politik Band 4

Herausgegeben von den Professoren

Dr. Ossip K. Flechtheim und Dr. Otto Heinrich von der Gablentz in Verbindung mit Professor Dr. Hans Reif im Auftrage des

Otto-Suhr-Instituts an der Freien Universität Berlin (vormals Deutsche Hochschule für Politik)

Redaktion: Ossip K. Flechtheim

Sir Ivor W. Jennings/Gerhard A. Ritter

\section{Das britische Regierungssystem}


Sir Ivor W. Jennings

Gerhard A. Ritter

\section{Das britische Regierungssystem}

Leitfaden und Quellenbuch

Zweite,

überarbeitete und erweiterte Auflage 
Ins Deutsche übersetzt

von Gerd Höfinghoff, Oskar Kruß und Gerbard A. Ritter

ISBN 978-3-663-00236-9 ISBN 978-3-663-02149-0 (eBook)

DOI 10.1007/978-3-663-02149-0

Verlags-Nr. 053104

(c) 1958 by Springer Fachmedien Wiesbaden

Ursprünglich erschienen bei Westdeutscher Verlag GmbH, Köln und Opladen 1958

Softcover reprint of the hardcover 2nd edition 1958 
Umfang und Art der Regierungsfunktionen haben sich in den letzten 50 Jahren gewaltig geändert. Früher war die Regierungstätigkeit hauptsächlich regulierend und negativ. Hauptaufgabe der Regierung war (abgesehen von der Verteidigung), den Ring freizuhalten und für die Beachtung der Grundregeln der Fairneß zu sorgen, während die privaten Interessen sich selbst frei zur Geltung brachten. Heute betrifft die Regierungstätigkeit weitgehend die Verwaltung der sozialen Dienste; sie ist positiv in einem neuen Sinn geworden. Noch vor einem Jahrhundert trat der Staat vor allem als Polizist, Soldat und Richter auf. Heute fungiert der Staat daneben als Arzt, Krankenschwester, Lehrer, Versicherungsunternehmer, Bauunternehmer, Gesundheitsinspektor, Chemiker, Eisenbahnkontrolleur, Unternehmer für die Gas-, Wasser- und Elektrizitätsversorgung, Stadtplaner, Verteiler von Renten, als Transportunternehmer, Organisator von Krankenhäusern, als Straßenbauer und in einer großen Anzahl anderer Funktionen.

Aus einem Memorandum von W. A. Robson für das Committee on Ministers' Powers, 1929-32 
Für die Erlaubnis, Auszüge aus Werken zu drucken, die noch unter Copyright stehen, dankt der Herausgeber den folgenden Verlagen:

C. H. Beck'sche Verlagsbuchhandlung (Morrison: Regierung und Parlament in England). Europa-Verlag Zürich A. G. (Winston S. Churchill: Reden, Bd. 4). Verlag Herbert Lang \& Cie. Bern (Magna Carta Libertatum von 1215). Cambridge University Press (Ivor Jennings: Cabinet Government, und Jennings: The British Constitution). The Clarendon Press Oxford (Butler: The Electoral System in Britain 1918-1951). Faber and Faber Ltd. (Bonham: The Middle Class Vote). The Hansard Society (Bailey: The Future of the House of Lords). John Murray (Publishers) Ltd. (Parker: Life of Sir Robert Peel). D. E. Butler and Macmillan \& Co. Ltd. (Butler: The British General Election of 1955 und D. E. Butler und A. King, The British General Election of 1966). Hutchinson \& Co. (Publishers) Ltd. (J. A. Spender and Cyril Asquith: Life of Herbert Henry Asquith, Lord Oxford and Asquith). Weidenfeld and Nicolson (Crick, The Reform of Parliament). Der Herausgeber dankt weiterhin: The Controller of Her Majesty's Stationery Office für die Erlaubnis des Abdrucks von Auszügen aus Gesetzen, Parlamentspapieren und Parlamentsdebatten. The Keeper of the Royal Archives at Windsor Castle für die Erlaubnis, Teile eines Memorandums von Lord Davidson vom Mai 1923 abzudrucken. The Executors of the late Viscount Esher für die Erlaubnis, ein Memorandum Viscount Eshers an König Georg V. (aus: Journals and Letters of Reginald Viscount Esher, Bd. 3) zu zitieren. The Royal Institut of Public Administration für den Abdruck eines Auszugs des Artikels von Sir Toby Low, The Select Committee on Nationalized Industries (in: Public Administration, Bd. 40, 1962). The National and English Review für den Artikel von Attlee und Boyle (Bd. 148, Jan. 1957). 


\section{Inhaltsverzeichnis}

Vorwort zur zweiten Auflage

\section{Leitfaden}

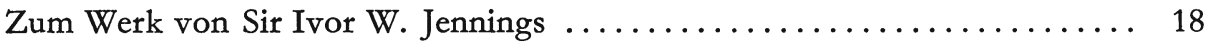

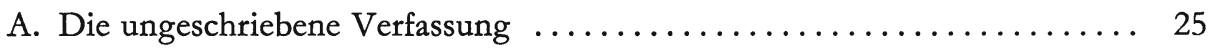

B. Die historische Tradition ............................. 29

C. Reiche und Territorien $\ldots \ldots \ldots \ldots \ldots \ldots \ldots \ldots \ldots \ldots \ldots \ldots \ldots \ldots \ldots \ldots \ldots, 40$

D. Die Monarchie ................................... 46

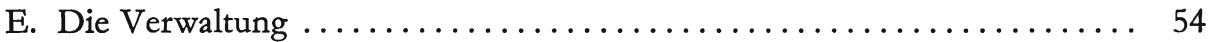

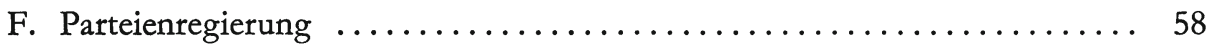

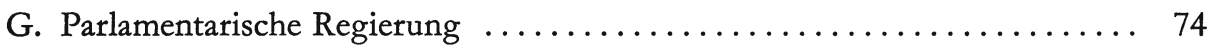

H. Das Finanzwesen ................................ 92

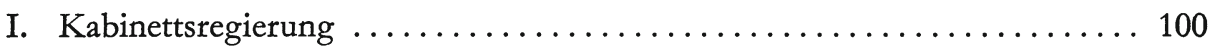

K. Die Herrschaft des Rechts .......................... 107

\section{Quellenbuch}

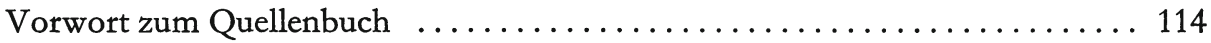

A. Die Herrschaft des Rechts ................................ 119

1. Magna Carta Libertatum, 1215 ........................ 119

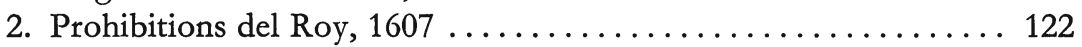

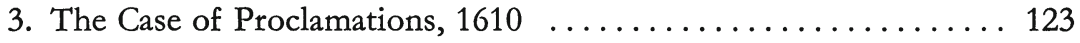

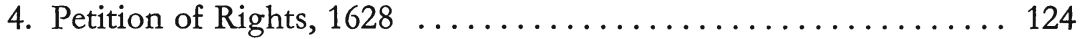

5. Habeas Corpus Amendment Act, 1679 .................. 125

6. Bill of Rights, 1689 ........................... 128

7. Die Herrschaft des Rechts und die Verwaltung ........... 134

a. Die Gerichte und die Verwaltung, 1765 ............... 134

b. Die rechtsprechenden Funktionen der Exekutive, 1957 ..... 138 
8. Die Herrschaft des Rechts und die Privilegien des Parlaments ... 142

a. Resolutionen des Unterhauses vom 31. 5. 1837 . . . . . . . . 143

b. Rechtsfall Stockdale contra Hansard, 1839 ........... 143

9. Das Recht des Bürgers und die Privilegien der Abgeordneten ... 146

B. Die Monarchie .................................. 149

1. Der Krönungseid .......................... 149

2. Die Regelung der Thronfolge $\ldots \ldots \ldots \ldots \ldots \ldots \ldots \ldots \ldots \ldots$

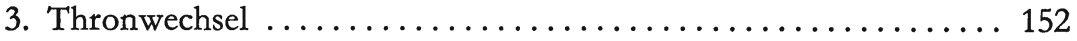

a. Der Thronwechsel und die Richter, $1760 \ldots \ldots \ldots \ldots \ldots \ldots 152$

b. Der Thronwechsel und das Parlament, 1867 ............ 152

c. Der Thronwechsel und die Ämterbesetzung, 1901 . . . . . . . . 153

4. Gesetz über Heiraten von Mitgliedern der Königlichen Familie,

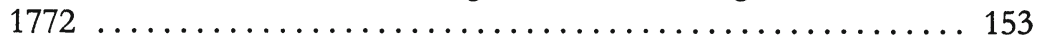

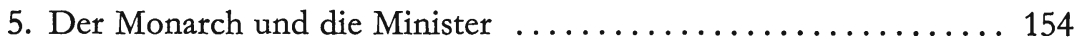

6. Der persönliche Einfluß des Monarchen .............. 155

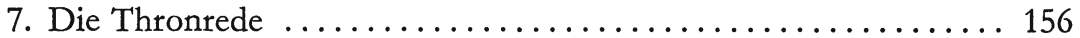

8. Das Vetorecht des Monarchen ..................... 159

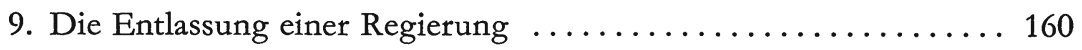

10. Die Auflösung des Parlaments ..................... 161

11. Die Krone und die Ernennung des Premierministers ........ 162

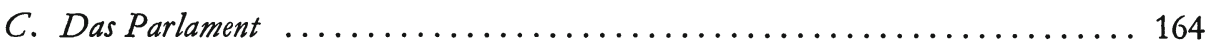

1. Die Souveränität des Parlaments .................... 164

2. Die Gerichte und die Gesetze des Parlaments ............ 165

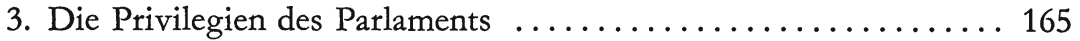

a. Die Petition des Sprechers des Unterhauses ............ 165

b. Das Unterhaus als Gerichtshof ................. 166

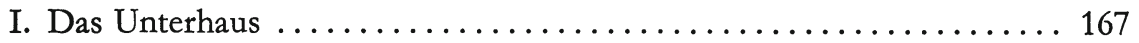

1. Die Finanzgewalt des Unterhauses ............... 167

a. Initiative im Unterhaus, $1661 \ldots \ldots \ldots \ldots \ldots \ldots \ldots \ldots \ldots \ldots$

b. Verbot der Veränderung von Bewilligungen der Commons

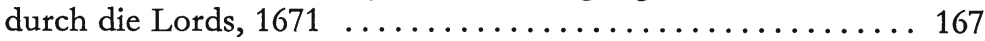

c. Die Rechte des Unterhauses in Finanzfragen, 1678 . . . . . . 168

2. Das Unterhaus und das House of Lords .............. 168

a. Das Parlamentsgesetz von 1911 ................. 168

b. Das Parlamentsgesetz von 1949 ................. 171

3. Das Unterhaus und die Regierung $\ldots \ldots \ldots \ldots \ldots \ldots \ldots \ldots \ldots$

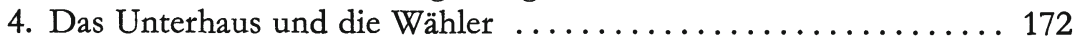

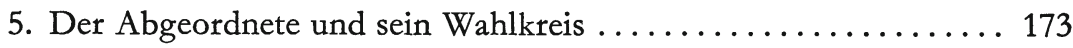

a. Die Theorie der Repräsentation ................ 173

b. Der Abgeordnete und seine lokale Parteiorganisation ...... 174

6. Der Abgeordnete und seine Fraktion ............... 178

7. Der Abgeordnete und die Vertretung von organisierten Interessen 179 
8. Die Opposition Ihrer Majestät . . . . . . . . . . . . . 180

a. Die Prägung des Ausdrucks . ................. 180

b. Die Opposition und die Verfassung $\ldots \ldots \ldots \ldots \ldots \ldots \ldots 181$

9. Die Neutralität des Sprechers $\ldots \ldots \ldots \ldots \ldots \ldots \ldots \ldots \ldots \ldots \ldots \ldots$

10. Die Arbeit des Unterhauses ..................... 182

11. Die Beschneidung der parlamentarischen Debatte .......... 186

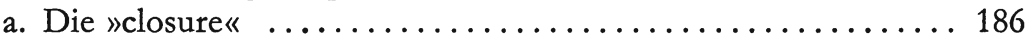

b. Die "guillotine« (Allocation of Time Order) .......... 187

c. Zeitplan für die Haushaltsdebatten . . . . . . . . . . . . 190

d. Die Auswahl von Zusatzanträgen durch den Sprecher ....... 192

12. Die Fragestunde des Unterhauses ................... 193

13. Ständige Ausschüsse (Standing Committees) . . . . . . . . . . 195

14. Spezialisierte Sachausschüsse . . . . . . . . . . . . . . . 197

15. Das Unterhaus und die nationalisierten Industrien . . . . . . . . . . 199

a. Parlamentarische Anfragen über nationalisierte Industrien ... 199

b. Methoden der parlamentarischen Kontrolle nationalisierter

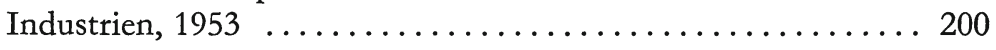

c. Regierungserklärung über die Bildung eines Ausschusses für die nationalisierten Industrien, 1954 . . . . . . . . . . 201

d. Die Einsetzung des Ausschusses, 1955 . . . . . . . . . . . 203

e. Die Arbeit des Ausschusses ................... 203

16. Das Amt des Parlamentsbeauftragten für die Verwaltung . . . . . 208

17. Das Parlament als Arbeitsstätte ...................... 212

18. Der Sitzungssaal des Unterhauses .................. 214

II. Das House of Lords . ....................... 217

1. Vorladung von Pairs zu einem neuen Parlament .......... 217

2. Das House of Lords als oberstes Appellationsgericht......... 218

3. Das House of Lords und die Mandatstheorie ............. 219

4. Die Aufgaben des House of Lords in der Gesetzgebung und Meinungsbildung ........................ 220

5. Das Problem der Reform des House of Lords ............. 221

a. Bericht von Viscount Bryce an den Premierminister, 1918 . . . 221

b. Die Konferenz der Parteiführer von 1948 . . . . . . . . . . . 223

c. Reformpläne der Labour Regierung, 1968 . . . . . . . . . . 224

6. Ansätze zur Reform des House of Lords . . . . . . . . . . . . . . 226

a. Die Ernennung von Pairs auf Lebenszeit ............. 226

b. Die Ausschaltung politisch inaktiver Pairs ............ 226

7. Der Peerage Act von 1963 ..................... 227

8. Die Zusammensetzung des House of Lords, 1968 ......... 230

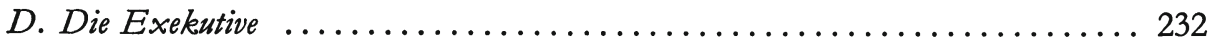

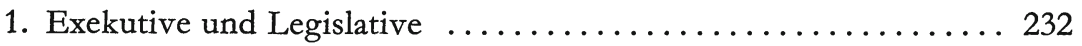

2. Das Recht der Exekutive zum Abschluß von Staatsverträgen .... 232 


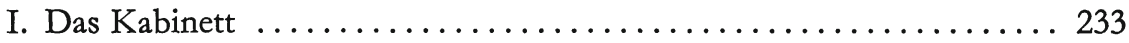

1. Kollektive Verantwortlichkeit des Kabinetts .............. 233

2. Der Premierminister .......................... 234

a. Der Premierminister und das Kabinett ............... 235

b. Der Premierminister und sein Außenminister .......... 236

c. Der Premierminister und die Wähler ................ 237

3. Die Macht des Kabinetts ......................... 238

4. Die Funktionen des Kabinetts .................... 238

5. Das Gesetz über die Minister der Krone von 1937 ......... 239

6. Die Zusammensetzung der Regierung Wilson, 1969 .......... 241

7. Das Anwachsen der Regierung, 1900-1968 ............... 248

8. Die Kabinettsmaschinerie ......................... 249

a. Das Kabinett vor 1914 ............................ 249

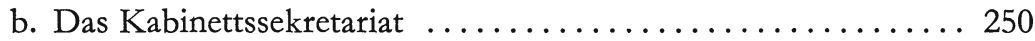

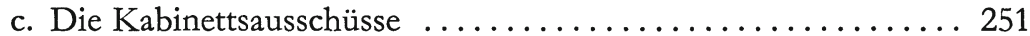

9. Die Koordinierung der Verteidigungspolitik ............ 252

10. Der Staat und die britische Wirtschaft ................. 257

a. Das National Economic Development Council ........... 257

b. Die Errichtung des National Board for Prices and Incomes ... 260

c. Die Industrial Reorganisation Corporation ............ 264

d. Die Commission on Industrial Relations $\ldots \ldots \ldots \ldots \ldots \ldots . \ldots 267$

II. Die Verwaltung und nationalisierten Wirtschaftszweige . . . . . . . . 269

1. Veränderungen der Struktur der Zentralverwaltung ......... 269

2. Die öffentlichen Körperschaften .................... 271

a. Die Errichtung des National Coal Board .............. 271

b. Die Nationalisierung der Eisen- und Stahlindustrie ........ 275

c. Die Elektrizität erzeugende Industrie und der zuständige Minister 280

d. Die nationalisierten Industrien als Wirtschaftsunternehmen ... 282

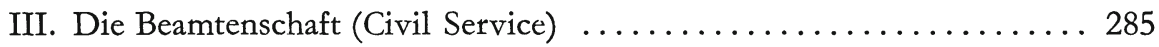

1. Die Rekrutierung der Beamtenschaft ................. 285

a. Die Civil Service Commission und die Auswahl der Bewerber . 285

b. Die Rekrutierung des Nachwuchses für die höhere Beamtenschaft .................................... 289

2. Verhaltensgrundsätze für Beamte ................. 290

3. Der Beamte und sein Minister ....................... 291

4. Der Beamte und die Politik ........................... 294

a. Der Bericht des Masterman-Ausschusses, 1949 .......... 294

b. Die Gruppe der Beamten mit beschränkter politischer Betätigungsfreiheit . .............................. 298

5. Umfang und Struktur des Civil Service ................. 300

a. Die Beamtenklassen .......................... 300

b. Die Beamtenschaft der einzelnen Regierungsbehörden ....... 301

c. Arbeiter im Beamtenverhältnis(Industrial Staff in the CivilService) 302 
6. Die Veränderungen der internationalen Lage und die neuen Aufgaben der britischen Vertretungen in Übersee . . . . . . . . . . 303

7. Das Problem der Reform des britischen Civil Service ........ 311

a. Der Bericht des Fulton-Ausschusses, $1968 \ldots \ldots \ldots \ldots \ldots \ldots 311$

b. Erklärung Premierminister Wilsons zu den Empfehlungen des Fulton-Ausschusses

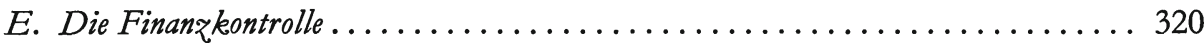

1. Die Grundsätze und die Praxis der Kontrolle öffentlicher Ausgaben 320

2. Die Finanzkontrolle des Unterhauses ............... 325

a. Die Initiative der Krone bei der Bewilligung von Geldmitteln . 326

b. Die Kontrollmaschinerie .................... 326

c. Der Wandel der Arbeit des Rechnungsprüfungsausschusses . . 329

F. Die Kommunalverwaltung.......................... 330

1. Die Struktur der englischen Kommunalverwaltung ........ 330

a. Die gesetzlichen Grundlagen ................ 330

b. Die Grenzen der kommunalen Körperschaften ........... 333

c. Der Plan zur Reform ....................... 337

2. Probleme der inneren Organisation der englischen Kommunal-

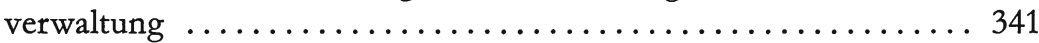

3. Die Kommunalverwaltung von Groß-London ............. 345

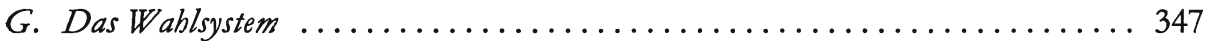

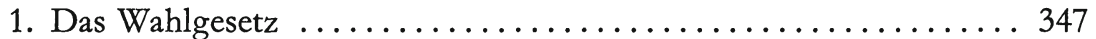

2. Die Festlegung der Wahlkreise ................... 351

3. Die Arbeitsweise einer Boundary commission ............. 355

4. Die Wahlkreise mit unsicheren Mehrheitsverhältnissen ........ 356

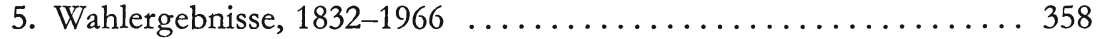

6. Wählerstimmen und Unterhaussitze, 1922-1966 . . . . . . . . . 359

7. Die Wahlergebnisse von 1966 ..................... 359

H. Die Organe der öffentlichen Meinungsbildung ................. 362

I. Rundfunk und Fernsehen ..................... 362

1. Die verfassungsmäßige Stellung von Rundfunk und Fernsehen . . 362

2. Der Einfluß des Fernsehens auf die Gesellschaft und das Problem der Programmgestaltung . . . . . ................. 364

3. Politische Sendungen .......................... 367

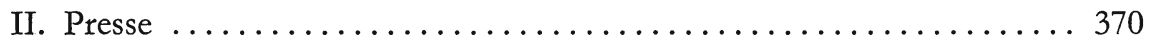

1. Der Einfluß der Presse und die Gefahr der Besitzkonzentration . . 370

2. Die vier führenden Pressegruppen, $1965 \ldots \ldots \ldots \ldots \ldots \ldots \ldots 373$

3. Die Kontrolle von Zeitungsverschmelzungen ........... 373

4. Die Beteiligung von Presseunternehmungen an Fernsehproduktions-

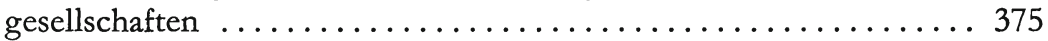


I. Die politischen Parteien ................................... 381

I. Die Labour Party ............................... 381

1. Verfassung und Geschäftsordnung der Labour Party, 1968 ...... 381

2. Die Pflichten der Mitglieder der Unterhausfraktion der Labour Party 391

3. Das Führungsgremium der Parlamentsfraktion der Labour Party in Zeiten der Opposition ........................... 393

4. Die Organisation der Parlamentsfraktion der Labour Party ...... 394

5. Finanz- und Organisationsprobleme der Labour Party ......... 395

6. Die Auswahl von Kandidaten für Parlamentswahlen ......... 401

7. Der Nationalrat der Arbeiterbewegung ................ 402

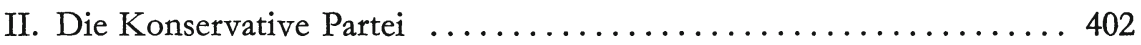

1. Die Organisation der Konservativen Partei ............. 402

2. Die Parlamentsfraktion der Konservativen Partei ........... 407

3. Der Führer der Konservativen Partei .................. 408

a. Die Wahl des Parteiführers ..................... 408

b. Wechsel der Führerschaft der Partei ................ 410

4. Das konservative Schattenkabinett und die Hauptsprecher der Konservativen Partei .......................... 412

5. Die Landesorganisation der Konservativen .............. 413

6. Die Wahlkreisorganisation der Konservativen ............. 414

7. Organisations- und Finanzfragen der Konservativen ......... 416

8. Die Auswahl konservativer Parlamentskandidaten in England und Wales .................................. 418

III. Die Parlamentskandidaten und die Wähler ............... 421

1. Schulbildung, Beruf und Religion der Konservativen und Labour Kandidaten bei der Parlamentswahl von 1966 ............. 421

2. Die Sozialstruktur der Wählerschaft der Konservativen und der Labour Party, 1945-1951 ........................... 424

J. Die Interessenverbände ............................ 428

1. Die Stellung der Interessengruppen im Regierungssystem des Landes 428

2. Gewerkschaften und Arbeitgeberorganisationen ............ 431

3. Die Gewerkschaften und die Politik ................. 432

4. Die Gewerkschaften und die Labour Party ............... 435

5. Das Problem der Reform der britischen Gewerkschaftsbewegung . 436

6. Die Confederation of British Industry $\ldots \ldots \ldots \ldots \ldots \ldots \ldots \ldots .440$

a. Ziele, Rechte und Grundsätze der Confederation ........... 440

b. Die Confederation und die Regierung $\ldots \ldots \ldots \ldots \ldots \ldots . . .441$

c. Die Öffentlichkeitsarbeit der Confederation ............. 442

7. Der Bauernverband und die Agrargesetzgebung ........... 443

Anbang

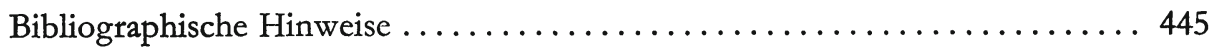




\section{Vorwort zur zweiten Auflage}

Die Bearbeitung der vorliegenden Neuauflage stellte den Herausgeber vor große Schwierigkeiten. Bereits eine kursorische Durchsicht der in den letzten zwölf Jahren erschienenen Quellen und Darstellungen zum britischen Regierungssystem zeigte, daß die politischen, wirtschaftlichen und gesellschaftlichen Institutionen des Landes und die ihre Arbeitsweise bestimmenden Konventionen und Vorstellungen in wesentlichen Punkten verändert wurden bzw. noch im Prozeß der Umbildung begriffen sind. Die Bearbeitung konnte sich daher nicht auf einige Ergänzungen beschränken, sondern mußte versuchen, sowohl eine umfassende Bestandsaufnahme des gegenwärtigen politischen Systems Großbritanniens zu leisten als auch den Prozeß der ständigen Veränderung der Verfassungsverhältnisse durch die Andeutung der Probleme, die z. Z. im Mittelpunkt der Diskussion stehen, zu verdeutlichen.

Durch den Tod von Sir Ivor Jennings im Dezember 1965 entfiel die Möglichkeit einer Neubearbeitung des Leitfadens durch den Verfasser selbst. In dieser Situation hat sich der Bearbeiter gegen eine völlige Neufassung des Leitfadens und für eine die Grundsubstanz des Werkes nicht antastende Überarbeitung entschlossen, in der lediglich die seit dem Abschluß des Manuskripts Ende 1954 eingetretenen faktischen Änderungen in Aufbau und Arbeitsweise des Regierungssystems durch Korrektur des Textes berücksichtigt und neue Entwicklungen in den behandelten Fragenkreisen kurz ergänzt wurden *.

Insbesondere wurde keine Auseinandersetzung mit den Meinungen und Interpretationen von Jennings, mit denen der Bearbeiter keineswegs immer übereinstimmt, versucht. Auch hat der Bearbeiter darauf verzichtet, im Leitfaden eine eigene Darstellung der von Jennings ausgeklammerten Sachgebiete zu geben.

Im Quellenbuch dagegen sind auf Grund einer systematischen Durchsicht und Auswertung der in den letzten zwölf Jahren von Regierung, Parlament, Parteien sowie Interessenverbänden publizierten Dokumente die eingetretenen Veränderungen möglichst vollständig berücksichtigt und in relativ ausführlichen Einleitungen zu den einzelnen Quellenstücken in ihrer Bedeutung skizziert worden. Obwohl eine Reihe der in der ersten Auflage abgedruckten Stücke weggelassen werden konnte, ist der Gesamtumfang des Quellenwerkes um erheblich mehr als die Hälfte erweitert worden. Das Quellenbuch ist so nicht als bloße Illustration zum Leitfaden gedacht, sondern soll eine selbständige Einführung in das gegenwärtige britische Regierungssystem und seine Grundprobleme an Hand der ausgewählten Texte bieten.

* Angesichts der Fülle der kleineren Korrekturen und Ergänzungen hätte eine Unterscheidung der von Jennings und der vom Bearbeiter stammenden Teile des Leitfadens die Lesbarkeit des Bandes so stark beeinträchtigt, daß darauf verzichtet werden mußte. Sämtliche Anmerkungen im Leitfaden stammen vom Bearbeiter. 
Die Veränderungen und Erweiterungen gegenüber der ersten Auflage können hier nur in einigen Stichworten angedeutet werden. Sie berühren die Schaffung neuer Institutionen zur Beaufsichtigung der rechtsprechenden Funktionen der Exekutive, die Einengung des bisherigen Ermessensspielraums des Monarchen bei der Ernennung des Premierministers, die Spannung zwischen den Privilegien der Abgeordneten und den Rechten der Bürger, die Einschränkung der Handlungsfreiheit der Abgeordneten durch den Druck ihrer lokalen Parteiorganisationen, die verschiedenen Aspekte einer Reform der Arbeit des Unterhauses, die auch in Deutschland vielerörterte Schaffung des Amtes eines Parlamentsbeauftragen für die Verwaltung nach skandinavischem Modell sowie Zusammensetzung, Macht und Funktionen des Oberhauses.

Eine weitere Quelle zeigt die stark anwachsende Zahl der Regierungsmitglieder in den letzten Jahrzehnten, die eine erhebliche Erweiterung der auch in anderen Bereichen bedeutenden Patronagemacht des Premierministers bewirkte. Die Hoffnung auf Förderung ihrer politischen Karriere bindet die Abgeordneten an den Premierminister und seine Helfer und trägt entscheidend zur Disziplinierung der Parlamentsfraktion - vor allem in der Labour Party - bei. Die außerordentliche Schwierigkeit, einen Premierminister, der nicht freiwillig zurücktritt, zur Aufgabe seiner Position zu zwingen, ohne gleichzeitig die Partei zu spalten und damit den fast sicheren Verlust der nächsten Wahlen zu riskieren, ist heute einer der neuralgischen Punkte der britischen Verfassung. Die Stärke der Stellung des Premierministers, die die des Bundeskanzlers übertrifft, hängt dabei nicht nur mit seinen Machtfunktionen als Regierungschef, sondern auch mit dem plebiszitären Charakter der Wahl als einer Entscheidung zwischen zwei Kandidaten für den Posten des Premierministers und dem Fehlen mächtiger, regionaler Parteiorganisationen, die in einem föderalistisch strukturierten Staat wie der Bundesrepublik zur Hausmacht potentieller Rivalen werden können, zusammen.

Angesichts der Bedeutung der öffentlichen Meinung für den Prozeß der Willensbildung in der modernen Demokratie werden in einem neuen Kapitel die verfassungsmäßige Stellung von Rundfunk und Fernsehen, die Regeln zur Sicherung ihrer parteipolitischer Neutralität sowie das auch in der Bundesrepublik in den letzten Jahren vielerörterte Problem, eine die freie Meinungsbildung gefährdende Besitzkonzentration im Pressewesen $\mathrm{zu}$ verhindern, behandelt. Das Kapitel über die Parteien wurde durch Quellen über die Auswahl der parlamentarischen Kandidaten, die Finanz- und Organisationprobleme der beiden Hauptparteien und die Demokratisierung der Wahl des konservativen Parteiführers ergänzt. Bei der Auswahl neuer Quellenstücke über die Interessenverbände kam es dem Herausgeber vor allem darauf an, die starke Stellung der großen Organisationen der Arbeitnehmer und Arbeitgeber im System der britischen Politik und Regierung zu verdeutlichen.

Den wichtigsten Grund für die Veränderung der Verfassungsinstitutionen und des Selbstverständnisses der das Regierungssystem tragenden Kräfte im letzten Jahrzehnt müssen wir im wirtschaftlichen und finanziellen Bereich suchen. Die ungenügende Leistungsfähigkeit der britischen Wirtschaft und vor allem die mangelnde Dynamik ihres Wachstums sowie die ständigen schweren Krisen der Zahlungsbilanz führten in Großbritannien zu immer erneuten Versuchen, durch grundlegende Reformen der alten und die Schaffung neuer Institutionen sowie die Planung der gesellschaftlichen und wirtschaftlichen Entwicklung die Ursachen der wirtschaftlichen Schwächen zu beseitigen 
und mit dem drängenden Problem der Modernisierung des Landes fertigzuwerden. Eine relativ große Zahl der hier abgedruckten Quellen zeigt die Auswirkungen dieser Bemühungen auf das Regierungssystem: Die Reform des bestehenden Systems der Kontrolle der öffentlichen Ausgaben durch die Einführung einer mittelfristigen Finanzplanung; die Anerkennung neuer Grundsätze der staatlichen Ausgabenpolitik; die stärkere Entwicklung der Wirtschaftsplanung durch die Schaffung eines National Economic Development Council und den Ausbau der Planungsabteilungen in den Ministerien; die Entwicklung einer staatlichen Institution zur Kontrolle der Preis- und Lohnentwicklung; die Bildung von Organisationen zur (auch finanziellen) Förderung des Konzentrationsprozesses als eines Mittels zur Rationalisierung sowohl der britischen Industrie als auch der zersplitterten und in Organisation und Management veralteten britischen Gewerkschaftsbewegung; das verstärkte Bemühen um die Steigerung der wirtschaftlichen Leistungsfähigkeit der nationalisierten Industrien.

Die durch die schwierige Situation der britischen Wirtschaft bewirkten Anstöße zur Reform gingen aber weit über den engeren Bereich der Wirtschafts- und Finanzfragen hinaus. Sie berühren die von der Regierung bereits eingeleiteten Bestrebungen zur grundlegenden Veränderung von Struktur und Charakter der britischen Beamtenschaft durch die Abschaffung der Gliederung in einzelne Beamtenklassen, die Förderung des Spezialistentums, die Einstellung von aus Wirtschaft, Wissenschaft und Technik kommenden Außenseitern sowie die Betonung der Verwandtschaft der Aufgaben der Beamten eines die Wirtschaft regulierenden und eine Fülle von sozialen Leistungen erbringenden Staates mit denen des Personals von Wirtschaftsunternehmungen. Auch für den diplomatischen Dienst wird die Abkehr von alten Traditionen und die zunehmende Konzentration seiner Arbeit auf die Unterstützung des britischen Außenhandels, ohne dessen Verstärkung die internationale Position Großbritanniens weiter geschwächt würde, gefordert. Selbst die innerbritische Diskussion über die Neuorganisation des traditionellen Systems der Kommunalverwaltung hat ihre tieferen Ursachen in den schweren Hindernissen, die die bisherige scharfe Trennung von Stadt und Land und die mangelnde Leistungsfähigkeit der kleineren Verwaltungseinheiten der wirtschaftlichen Entwicklung des Landes in den Weg legten.

Insgesamt kann man die britische Diskussion der letzten zwölf Jahre über die Reform des Regierungssystems dahingehend zusammenfassen, daß in eindrucksvollen und oft schonungslosen Analysen die Schwächen des bisherigen Systems und der ihm zugrunde liegenden Prinzipien diagnostiziert werden, daß vielfach auch bereits eine Therapie zur Heilung dieser Schwächen vorgeschlagen oder eingeleitet wurde, daß aber noch keineswegs klar ist, ob der Patient die ihm verordnete, oft bittere Medizin der Abkehr von alten, historisch bewährten Institutionen und Gewohnheiten auch schlucken wird. Die politischen Initiativen zur Anpassung Großbritanniens an die sich rapide wandelnden Bedingungen eines modernen Staates haben bisher noch keinen wirklich nachhaltigen Einfluß auf die Steigerung der wirtschaftlichen Leistungsfähigkeit und die gesellschaftliche Modernisierung gehabt. Immerhin ist es noch zu früh, um die endgültigen Konsequenzen vieler der unter dem Druck der wirtschaftlichen Krise bereits eingeleiteten Veränderungen abzuschätzen oder gar die Chancen zur Verwirklichung der in den letzten Jahren lancierten, teilweise sehr weitgehenden Reformvorschläge und deren etwaige praktische Folgen zu beurteilen. 
Im Vergleich zu den ständigen, wenn auch noch nicht wirklich erfolgreichen Bemühungen um Steigerung seiner Effizienz ist ein anderes Grundproblem des sozialen Leistungsstaates in einer hochtechnisierten Industriegesellschaft - eine parallel zur zunehmenden Komplexität von Verwaltung und Politik einhergehende Verbesserung und Verfeinerung der Methoden der demokratischen Kontrolle - von Großbritannien trotz aller Diskussion um die Parlamentsreform nur sehr zögernd in Angriff genommen worden. Großbritannien steht wie jede moderne Industriegesellschaft vor der Aufgabe, ohne Beeinträchtigung der Funktions- und Leistungsfähigkeit dieser Gesellschaft, die die Voraussetzung für wirtschaftlichen und sozialen Fortschritt bedeutet, neue, wirksame Methoden ihrer Kontrolle zu entwickeln, die den Sachverstand nicht ausschließen, sondern ihn politischen Zielsetzungen unterordnen.

Eine ausführliche, kritische, die Literatur zu den einzelnen Sachgebieten einordnende Bibliographie schließt das Werk ab. Sie ist nicht nur als Orientierungsmittel für Studenten, sondern auch als Arbeitsinstrument für weiterführende Forschungen zum britischen Regierungssystem gedacht.

Der besondere Reiz des Studiums des britischen Regierungssystems für den Leser in der Bundesrepublik liegt in der Ählichkeit der britischen und deutschen Verfassungsstruktur und der nicht zuletzt dadurch bedingten Parallelität vieler Verfassungsprobleme. Auf der Suche nach adäquaten Lösungen dieser Probleme, wie der Frage der Pressekonzentration oder des zu geringen Einflusses des Parlaments auf die öffentliche Meinungsbildung, wird man daher immer wieder auf das britische Modell schauen, wie auch umgekehrt Einrichtungen der deutschen Demokratie, wie das Ausschußwesen des Bundestages oder die Struktur der Gewerkschaftsbewegung, einige Grundsätze in den Beziehungen der Sozialpartner oder die Verteilung der staatlichen Machtkompetenzen zwischen der Zentralgewalt und föderalen wie kommunalen Machtträgern von einzelnen britischen Politikern und Politikwissenschaftlern zur Begründung von Reformvorschlägen für Großbritannien herangezogen wurden. Ein derartiger Austausch von Anregungen kann aber nur dann sinnvoll sein, wenn man den Rahmen berïcksichtigt, in den die einzelnen politischen Institutionen eingeordnet sind und die Bedingungen kennt, unter denen sie operieren. $\mathrm{Zu}$ einem derart vertieften, auch die eigene Reformdiskussion befruchtenden Verständnis des britischen Regierungssystems beizutragen, ist die Aufgabe dieses Bandes.

Das Manuskript wurde im Februar 1969 abgeschlossen. Von späteren Veränderungen konnte im Fahnendruck nur noch der im Juni 1969 vorgelegte Plan zur Reform der Struktur der englischen Kommunalverwaltung (S. 337 ff.) berücksichtigt werden.

Für ihre Hilfe bei der Beschaffung des Quellenmaterials oder dem Lesen der Korrekturen habe ich Agnes Ahlers, Dr. Jürgen Kocka, Marie-Luise Recker und Heide-Irene Windschiegl zu danken. 